\title{
Optical generation of quasisolitons in a single-component gas of neutral fermionic atoms
}

\author{
T. Karpiuk, ${ }^{1}$ M. Brewczyk, ${ }^{1}$ E. Dobrek ${ }^{2}$, M.A. Baranov ${ }^{2}$, M. Lewenstein ${ }^{2}$, and K. Rzążewski ${ }^{3}$ \\ 1 Uniwersytet w Biatymstoku, ul. Lipowa 41, 15-424 Biatystok, Poland \\ 2 Institut für Theoretische Physic, Universität Hannover, 30167 Hannover, Germany \\ ${ }^{3}$ Centrum Fizyki Teoretycznej PAN and College of Science, Al. Lotników 32/46, 02-668 Warsaw, Poland
}

(Dated: November 28, 2018)

\begin{abstract}
We analyze the generation of soliton-like solutions in a single-component Fermi gas of neutral atoms at zero and finite temperatures with the phase imprinting method. By using both the numerical and analytical calculations, we find the conditions when the quasisolitons, which apparently resamble the properties of solitons in non-linear integrable equations, do exist in a non-interacting Fermi gas. We present the results for both spatially homogeneous and trapped cases, and emphasize the importance of the Fermi statistics and the absence of the interaction for the existence of such solutions.
\end{abstract}

PACS number(s): 05.30.Fk, 03.75.Fi

\section{INTRODUCTION}

Recent pioneering work by DeMarco and Jin [1] on realization of quantum degeneracy in a trapped gas of fermionic ${ }^{40} \mathrm{~K}$ atoms has triggered the interest in properties of cold fermions. As opposed to bosons, fermions cannot occupy the same quantum state, i.e., they obey the Pauli exclusion principle. Since at low temperatures only the $s$-wave scattering gets important, the spinpolarized fermions stop to collide at lower temperatures and rethermalization process necessary for evaporation breaks down. This difficulty has been overcome by trapping and cooling the fermions in two hyperfine states, enabling in this way the evaporation by collisions between atoms in a different spin states [1, 2]. Other groups have achieved quantum degeneracy in a gas of ${ }^{6} \mathrm{Li}$ atoms by sympathetic cooling of a mixture of bosonic $\left({ }^{7} \mathrm{Li}\right)$ and fermionic $\left({ }^{6} \mathrm{Li}\right)$ atoms reaching the temperatures down to $0.2 T_{F}$ [3, 4.

Despite of experimental difficulties in achieving the strong quantum degeneracy in fermionic gases, some theoretical work on degenerate Fermi gases in the normal phase at zero and finite temperatures has already been done. The static properties of one- and two-component system were analysed in Refs. [5], [6] and [7]. Collective excitations in degenerate Fermi gases in the normal phase were discussed within the hydrodynamic approximation in Ref. [8] and on the basis of the sum rules in Ref. [9]. In the absence of $s$-wave collisions other forces, like for instance dipole-dipole interaction, start to play role. The fermionic dipoles have been investigated recently, including the analysis of stability conditions 10. Another aspect of dynamic behavior of a degenerate Fermi gas is studied in Ref. [11] where the question about the possibility of generation of solitons and vortices in a normal state of non-interacting Fermi gas is asked. It is shown by solving the many-body Schrödinger equation that the phase imprinting method is capable of generating the solitons and vortices in such a system. An alternative method of generating such structures is discussed in Ref.
12.

So far, the phase imprinting method has been successfully applied to generate solitons in Bose-Einstein condensates [13, 14]. Although not realized experimentally yet, it offers also a rather rare opportunity of control of the generation of vortices [15, 16]. The method consists in passing an off-resonant laser pulse through the appropriately designed absorption plate followed by the impinging it on the atomic gas. The laser pulse is short and strong enough so that the atomic motion is negligible during the pulse and the basic effect of light is a generation of strong optical potential $V_{\text {opt }}(\vec{r}, t)$ due to the ac-Stark effect. This potential acts for a short time $\tau_{d u r}$, and essentially imprints a desired phase of atomic wave functions $\varphi(\vec{r})=\int_{0}^{\tau_{d u r}} V_{o p t}(\vec{r}, t) d t / \hbar$. After this, the density and the phase try to adopt to each other, which results in generation of structures like solitons and vortices, depending on the pattern written in the absorption plate.

In this paper we explore further the possibility of generating the quasisolitons in a gas of non-interacting Fermi atoms. According to the common knowledge regarding solitons, the nonlinearity of the problem plays essential role, since it cancels the dispersion and allows for a propagation of shape preserving pulses. So, at the first glance it is surprising that solitons can exist in a system of non-interacting particles described by the many-body Schrödinger equation, i.e., the linear equation. However, we show that, due to the Fermi statistics, there exists the regime of parameters where soliton-like solutions can exist in the non-interacting Fermi gas.

The paper is organized as follows. In Sec. II we solve the many-body Schrödinger equation for a system of non-interacting fermions undergoing the phase imprinting, leading to the generation of soliton-like structures. Both zero and finite temperatures are considered. Sec. III offers an analytical approach to the problem of propagation and decay of solitons, based on the Wigner function formalism, for a degenerate as well as a Boltzmann gas. In Sec. IV another approach based on analysis 
of density matrices, is followed in order to discuss the Thomas-Fermi approximation and to understand the role of dimensionality. Finally, we conclude in Sec. V.

\section{SOLUTION OF THE MANY-BODY SCHRÖDINGER EQUATION}

\section{A. Zero temperature}

At zero temperature the many-body wave function of a system of non-interacting particles (fermions) is given by the Slater determinant with the lowest available oneparticle orbitals occupied.

$$
\Psi\left(\vec{r}_{1}, \ldots, \vec{r}_{N}\right)=\frac{1}{\sqrt{N !}}\left|\begin{array}{ccc}
\varphi_{1}\left(\vec{r}_{1}\right) & \ldots & \varphi_{1}\left(\vec{r}_{N}\right) \\
\cdot & & \cdot \\
\cdot & & \cdot \\
\cdot & \cdot \\
\varphi_{N}\left(\vec{r}_{1}\right) & \ldots & \varphi_{N}\left(\vec{r}_{N}\right)
\end{array}\right| .
$$

The one-particle orbitals $\left(\varphi_{1}(\vec{r}), \ldots, \varphi_{N}(\vec{r})\right)$ are orthogonal and they undergo unitary evolution. Hence, their orthogonality is sustained, both during the phase imprinting and afterwords. Therefore, the one-particle density matrix is at any time given by the following formula

$$
\rho_{1}\left(\vec{r}^{\prime}, \vec{r}^{\prime \prime}, t\right)=\frac{1}{N} \sum_{i=1}^{N} \varphi_{i}\left(\vec{r}^{\prime}, t\right) \varphi_{i}^{*}\left(\vec{r}^{\prime \prime}, t\right),
$$

and its diagonal part is just the particle density.

Evolution of each single-particle orbital is obtained from the one-particle Schrödinger equation and can be split into two stages. First, the phase imprinting technique is used to disturb the system in a way of writing a desired phase on it. Assuming fast enough phase imprinting, the resulting wave function has the form (this procedure can be also performed numerically within the finite duration of the imprinting process):

$$
\varphi_{k}(\vec{r}) \longrightarrow \varphi_{k}^{\prime}(\vec{r})=\varphi_{k}(\vec{r}) \exp (i \phi(x)),
$$

for $k=1, \ldots, N$, where $\phi(x)$ is the phase imprinted on each atom. Throughout this paper we deal with the generation of quasisolitons, we assume that the imprinted phase depends only on a single coordinate, say ' $x$ ', and utilize $\phi(x) \sim\left(1+\tanh \left(x / d_{\text {phase }}\right)\right)$. We consider a Fermi gas in a three-dimensional box or harmonic trap characterized by the frequencies $\omega_{x}, \omega_{y}$, and $\omega_{z}$. Both are separable in Cartesian coordinates. The one-particle orbitals $\varphi_{k}(\vec{r})$ are taken as a product $\varphi_{n_{x}}^{(1)}(x) \varphi_{n_{y}}^{(2)}(y) \varphi_{n_{z}}^{(3)}(z)$ of eigenvectors of the Hamiltonians of one-dimensional boxes or harmonic oscillators.

In the second stage the system undergoes the free evolution in three-dimensional space. However, because the Hamiltonian separates in coordinates ' $x$ ', ' $y$ ', and ' $z$ ', the time propagation of each orbital can be easily reduced to

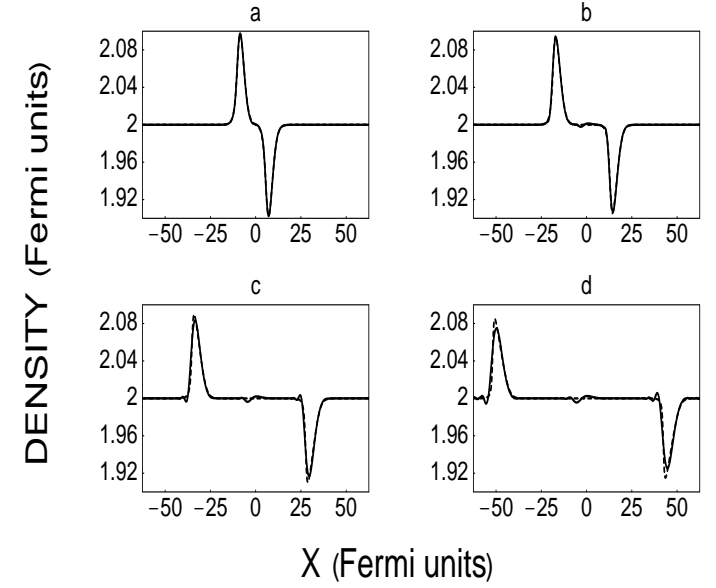

FIG. 1: Images of the fermionic density (normalized to the number of atoms) of $\mathrm{N}=500$ atoms after writing a single phase step of $1.0 \pi$ and the width $d_{\text {phase }}=2.5 \lambda_{F}$ onto a onedimensional non-interacting Fermi gas confined in a box at different times: (a) 24.7, (b) 49.3, (c) 98.7, and (d) 148.0 in Fermi units $\left(\hbar / \varepsilon_{F}\right)$. Each graph consists of two curves: one obtained based on the many-body Schrödinger equation (solid line) and the second coming from the one-dimensional Thomas-Fermi model (dashed line).

one-dimensional case:

$$
\begin{aligned}
\varphi_{k}(x, y, z, t) & =e^{-i \mathrm{E}_{\mathrm{n}_{y}} \mathrm{t} / \hbar} e^{-i \mathrm{E}_{\mathrm{n}_{\mathrm{z}}} \mathrm{t} / \hbar} \\
& \times \varphi_{n_{y}}^{(2)}(y, 0) \varphi_{n_{z}}^{(3)}(z, 0) \\
& \times \varphi_{n_{x}}^{(1)}(x, t)
\end{aligned}
$$

where

$$
\varphi_{n_{x}}^{(1)}(x, t)=e^{-i \mathrm{H}_{\mathrm{x}} \mathrm{t} / \hbar} \varphi_{n_{z}}^{(1)}(x, 0) e^{i \phi(x)}
$$

and $\mathrm{H}_{\mathrm{x}}$ is the Hamiltonian of one-dimensional box or harmonic oscillator. The diagonal part of one-particle density matrix is given then by the expression:

$$
\begin{gathered}
\rho(\vec{r}, t)=\frac{1}{\mathrm{~N}} \sum_{n_{x}, n_{y}, n_{z}}\left|\varphi_{n_{y}}^{(2)}(y, 0)\right|^{2}\left|\varphi_{n_{z}}^{(3)}(z, 0)\right|^{2} \\
\times\left|\varphi_{n_{x}}^{(1)}(x, t)\right|^{2} .
\end{gathered}
$$

In Fig. 1 we plotted the density profiles of a uniform system of 500 atoms after imprinting the phase designed in the form $\phi(x)=\phi_{0}\left(1+\tanh \left(x / d_{\text {phase }}\right)\right) / 2$. The characteristic length (the Fermi length) is given by the formula $\lambda_{F}=h / p_{F}=2 / N L$, where $p_{F}$ is the Fermi momentum and $L$ is the size of one-dimensional box. For the case of Fig. 1, the Fermi length equals $0.004 \mathrm{~L}$ and is 2.5 times smaller than the width characterising the jump of the imprinted phase. As discussed more precisely in Sec. III, the evolution of the density after phase imprinting depends strongly on the ratio $d_{\text {phase }} / \lambda_{F}$ between the width of the phase step and the Fermi length.

It is clear from Fig. 11 (as well as Fig. 2) that two quasisolitons (the bright and the dark one) are generated. 
TABLE I: Speeds of dark and bright quasisolitons (in units of speed of sound) generated in a one-dimensional homogeneous Fermi gas of 500 atoms after imprinting a single phase step of $\pi$ and various widths $d_{\text {phase }}$ (in units of the Fermi length).

\begin{tabular}{ccc}
\hline \hline$\frac{d_{\text {phase }}}{\lambda_{F}}$ & $\frac{v_{b}}{c}$ & $\frac{v_{d}}{c}$ \\
\hline 1.25 & -1.061 & +0.953 \\
2.50 & -1.044 & +0.958 \\
5.00 & -1.033 & +0.963 \\
12.5 & -1.014 & +0.981 \\
\hline \hline
\end{tabular}

They propagate in opposite directions with distinct velocities. We present in Table If the values of soliton's velocities for various widths of the phase steps. The speed of the bright quasisoliton is always higher than the speed of sound whereas dark quasisolitons move slower than the sound. However, increasing the ratio $d_{\text {phase }} / \lambda_{F}$ both velocities are getting closer to the speed of sound $\left(p_{F} / m\right.$ in one-dimensional space), reaching eventually (i.e., in the limit $d_{\text {phase }} \gg \lambda_{F}$ ) the value $p_{F} / m$ as shown in Secs. III and IV. In Sec. IV we also analyze the $d_{\text {phase }} \gg \lambda_{F}$ limit by decreasing $\lambda_{F}$ (increasing the number of atoms) and solving the equations of the Thomas-Fermi model.

Fig. 2 shows that going deeper into the regime $d_{\text {phase }} \gg \lambda_{F}$ the soliton structures become less pronounced. This again is explained in terms of Wigner function in Sec. III and based on Thomas-Fermi approach in Sec. IV. The condition $d_{\text {phase }} \gg \lambda_{F}$ means that the height (or depth) of solitons is getting smaller in comparison with unperturbed density. Going in opposite direction (i.e., $d_{\text {phase }} \lesssim \lambda_{F}$ ) results, as presented in Fig. 3, in a change of shape of the structures (dispersion) on a time scale of the order of $\hbar / \varepsilon_{F}$.

Another aspect of Fig. 11 is connected with the Thomas-Fermi approximation discussed in Sec. IV. Each frame of Fig. 1 shows, in fact, two curves; one of them (solid line) is obtained by solving the many-body Schrödinger equation, and the second (dashed line) is representing the Thomas-Fermi approach. Both curves match well which proves the validity of the ThomasFermi approximation in one-dimensional space for large enough number of atoms just as it happens in the case of a harmonic trap (see Fig. 1 in Ref. [11], where twoand three-dimensional cases are also discussed).

\section{B. Finite temperatures}

One way of including temperature effects on the soliton's generation and propagation is to allow for populating the single-particle states of higher energy than the Fermi energy. We have realized this by considering a grand canonical ensemble for a system of non-interacting fermions. We generate a number of many-particle configurations according to the Fermi-Dirac statistics, i.e., with the probability of populating a particular one-

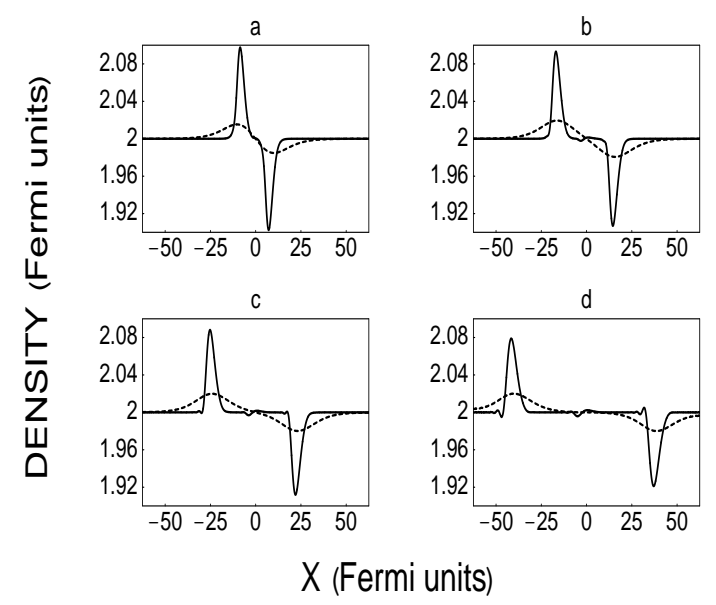

FIG. 2: Comparison of bright-dark soliton structures for various widths of imprinted phase: $2.5 \lambda_{F}$ (solid line) and $12.5 \lambda_{F}$ (dashed line). The snapshots are taken at times (a) 24.7, (b) 49.3, (c) 74.0, and (d) 123.3 in Fermi units. Other parameters are the same as in Fig. 11.

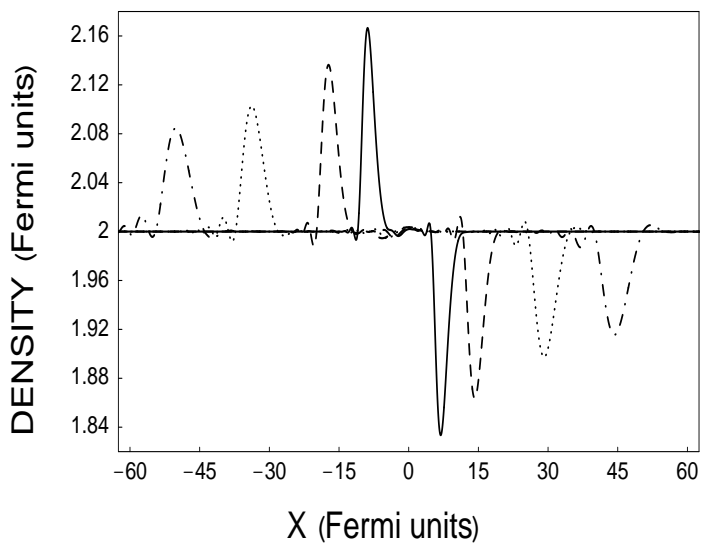

FIG. 3: Soliton-like structures in the regime where $d_{\text {phase }} \lesssim$ $\lambda_{F}$ (here, $\left.d_{\text {phase }}=1.25 \lambda_{F}\right)$. The snapshots are taken at times (a) 24.7 (solid line), (b) 49.3 (dashed line), (c) 98.7 (dotted line), and (d) 148.0 (dashed-dotted line) in Fermi units. Other parameters are the same as in Fig. 目.

particle state $\varphi_{k}$ given by $\left.\exp \left(\beta\left(\varepsilon_{k}-\mu\right)\right)+1\right)^{-1}$, where $\varepsilon_{k}$ is the state energy, $\beta$ determines the bath temperature (via $\beta=1 / k_{B} T$ ), and $\mu$ is the chemical potential (dependent on temperature in general). More precisely, for each one-particle state (unless the probability of populating this state is lower than $10^{-6}$ ) we generate a random number from the interval $[0,1]$. If the number is less than $\left.\exp \left(\beta\left(\varepsilon_{k}-\mu\right)\right)+1\right)^{-1}$, the one-particle state is populated, otherwise it is left empty. Here, $\varepsilon_{k}$ is the energy of the state under consideration, the chemical potential

$$
\mu=\varepsilon_{F}\left[1-\left(\pi^{2} / 3\right)\left(k_{B} T / \varepsilon_{F}\right)^{2}\right],
$$

and $\varepsilon_{F}$ equals the Fermi energy corresponding to the average number of atoms. The above formula for the chemical potential applies for the Fermi gas trapped in 
a harmonic potential for the temperatures up to $0.55 T_{F}$ [5].

In such a way, having given the temperature and the average number of atoms, the many-particle configurations are sampled. Then, for each many-particle configuration the phase imprinting is performed followed by a free evolution. Finally, the density is calculated as an average according to a grand canonical ensemble rules. We have investigated in this way the propagation of quasisolitons for temperatures up to $0.3 T_{F}$, already attainable in experiment 2 , 3 , 田.

In Fig. 4 we plot evolution of the averaged density of the Fermi gas confined in a one-dimensional harmonic trap of frequency $\omega$ after phase imprinting of a single step of $2 \pi$ and the width equal to 0.5 osc. units. $(\sqrt{\hbar /(m \omega)})$. Here, the chemical potential is given by the formula $\mu=\varepsilon_{F}+k_{B} T \log \left[1-\exp \left(-\varepsilon_{F} / k_{B} T\right)\right]$, which is a onedimensional counterpart of formula (4), the temperature equals $0.2 T_{F}$, and the averaging procedure is performed over 5000 many-body configurations. Both, bright and dark solitons are still present in the system exhibiting basically the same properties as at zero temperature. For higher temperatures (already $0.3 T_{F}$ ) the soliton structures start to get hidden within the noise originating in the averaging procedure (Fig. 5). However, one has to remember that rather a particular many-body configuration is realized in a single experiment, and not an ensemble of them. Finally, Fig. 6 gives an example that going from one - to three-dimensional space, predictions made based on lower dimensionality survive. Fig. 6 shows density profiles of fermionic gas trapped in a spherically symmetric potential (averaged over 1000 configurations at $0.1 T_{F}$ temperature) and can be compared with corresponding figure from Ref. [11] at zero temperature since the details of phase imprinting are the same.

\section{WIGNER FUNCTION APPROACH}

The Wigner function $w(x, p, t)$ is defined as

$w(x, p, t)=\int d y\left\langle\hat{\psi}^{\dagger}(x+y / 2, t) \hat{\psi}(x-y / 2, t)\right\rangle \exp (-i p y)$

where $\hat{\psi}^{\dagger}(x, t)$ and $\hat{\psi}(x, t)$ denote atomic creation and annihilation operators in the Heisenberg picture, respectively. It obeys the equation

$$
\left(\partial_{t}+\frac{p}{m} \partial_{x}-\partial_{x} V_{e x t}(x) \partial_{p}\right) w(x, p, t)=0,
$$

where $V_{\text {ext }}(x)$ is the external harmonic trap potential. It immediately follows from the definition, Eq. (5), that the density of particles at time $t$ is

$$
\rho(x, t)=\int \frac{d p}{2 \pi \hbar} w(x, p, t)
$$

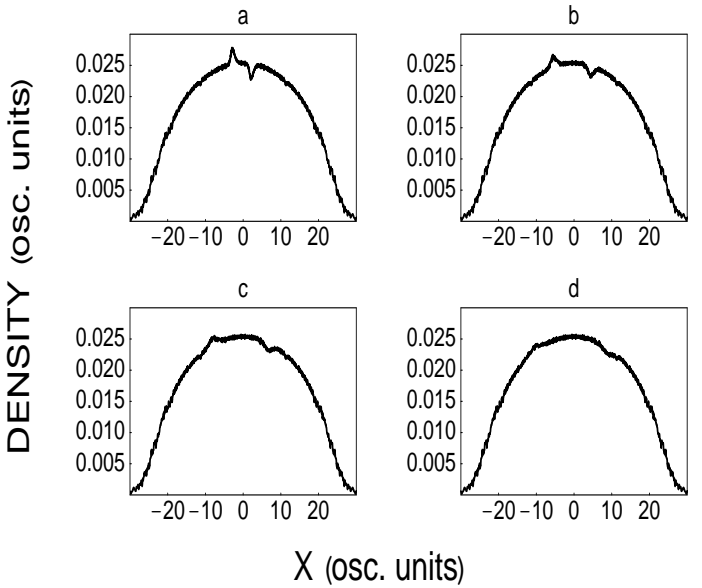

FIG. 4: Evolution of the density distribution of 300 (average number) Fermi atoms in one-dimensional harmonic trap at temperature $0.2 T_{F}$ after imprinting a single phase step of $2.0 \pi$ and $d_{\text {phase }}=0.5$ osc. units. The successive frames correspond to moments: (a) 0.1 , (b) 0.2 , (c) 0.3 , and (d) 0.4 in units of $1 / \omega$.

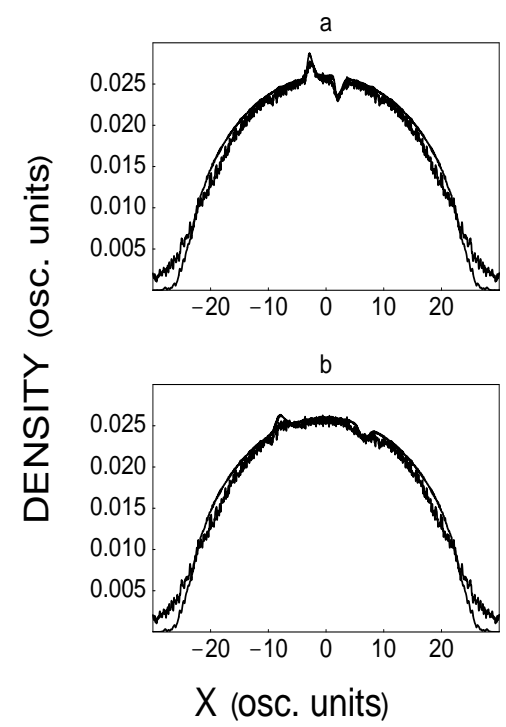

FIG. 5: Comparison of bright-dark soliton structures at different temperatures: $0.1 T_{F}$ (less rugged curve, averaged over 1000 configurations) and $0.3 T_{F}$ (more rugged curve, averaged over 10000 configurations). Frames (a) and (b) correspond to moments 0.1 and 0.3 in units of $1 / \omega$ respectively and other parameters are the same as in Fig. 4 .

The phase imprinting at $t=0$ modifies the Wigner function in the following way:

$$
\begin{aligned}
w_{0}(x, p, t & =0) \rightarrow \\
w_{0}(x, p)= & \int d y\left\langle\hat{\psi}^{\dagger}(x+y / 2, t=0) \hat{\psi}(x-y / 2, t=0)\right\rangle \\
& \times \exp (-i p y / \hbar-i \phi(x+y / 2)+i \phi(x-y / 2)),
\end{aligned}
$$




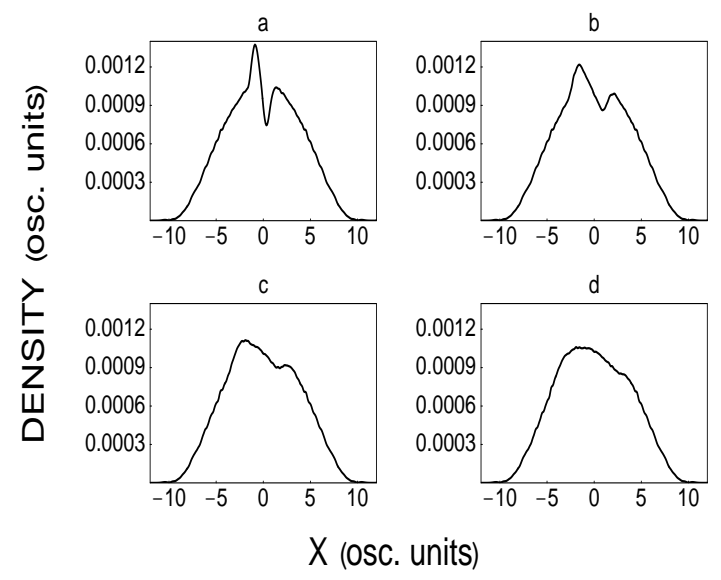

FIG. 6: Density profiles of non-interacting fermionic gas confined in three-dimensional spherically symmetric harmonic trap at different times: (a) 0.1 , (b) 0.2 , (c) 0.3 , and (d) 0.4 in units of $1 / \omega$ after writing a single phase step of $\phi_{0}=1.0 \pi$ and $\zeta=0.2$ osc. units. The average number of atoms $\mathrm{N}=10^{4}$, the temperature is equal to $0.1 T_{F}$, and 1000 configurations were used in the averaging procedure.

where $\phi(x)$ is the imprinted phase. The function $w_{0}(x, p)$ provides the initial condition for the dynamical evolution according to Eq. (6).

Let us consider first the spatially homogeneous case $\left(V_{e x t}(x)=0\right)$. Eq. (6) with the initial condition $w(x, p, t=0)=w_{0}(x, p)$ can be easily solved. The solution is

$$
w(x, p, t)=w_{0}(x-(p / m) t, p),
$$

and reflects the ballistic motion of particles in the absence of an external potential. By using the fact that in the spatially homogeneous case the correlation function $\left\langle\hat{\psi}^{\dagger}(x+y / 2, t=0) \hat{\psi}(x-y / 2, t=0)\right\rangle=\rho_{0}(y)$, i.e., it depends only on ' $y$ ', the expression for $w_{0}(x, p)$ can be rewritten in the form

$$
\begin{gathered}
w_{0}(x, p)=\int d y \rho_{0}(y) \exp (-i p y / \hbar-i \phi(x+y / 2) \\
+i \phi(x-y / 2)) .
\end{gathered}
$$

The above formulae have a general character, and are valid for both degenerate and non-degenerate (Boltzmann) Fermi gas. The difference between these two cases is only in the specific form of the function $\rho_{0}(y)$.

Usually in the experiments, the imprinted phase $\phi(x)$ is a function with transition regions of the size of the order of $d_{\text {phase }}$ between the regions with constant values of the phase. Depending on the ratio between $d_{\text {phase }}$ and the characteristic width of the function $\rho_{0}(y)$, one has completely different behavior of the solution Eq. (9) and, therefore, the density evolution. In the degenerate regime, the characteristic width of $\rho_{0}$ is given by the Fermi wavelength $\lambda_{F}$, which is inversely proportional to the Fermi momentum, $\lambda_{F}=h / p_{F}$. In the opposite case of a Boltzmann gas, the width is given by the thermal wavelength $\lambda_{T}=h / \sqrt{2 m T}$.

We first analyze the case where $d_{\text {phase }} \gg \lambda_{F}, \lambda_{T}$. Under this condition, one can expand the exponent in Eq. (10) in powers of $y$, and leave only the lowest, linear in $y$, contribution:

$$
\begin{aligned}
w_{0}(x, p) & \approx \int d y \rho_{0}(y) \exp \left(-i p y / \hbar-i \phi^{\prime}(x) y\right) \\
& =\widetilde{\rho}_{0}\left(p+\phi^{\prime}(x)\right)
\end{aligned}
$$

where $\widetilde{\rho}_{0}(p)$ is the Fourier transform of the function $\rho_{0}(y)$ and $\phi^{\prime}$ is the derivative of $\phi$. The density of the particles, Eq. (7), can then be written as

$$
\begin{aligned}
\rho(x, t) & =\int \frac{d p}{2 \pi \hbar} w_{0}(x-(p / m) t, p) \\
& \approx \int \frac{d p}{2 \pi \hbar} \widetilde{\rho}_{0}\left(p+\hbar \phi^{\prime}(x-(p / m) t)\right) .
\end{aligned}
$$

Keeping in mind that $\phi^{\prime} \lesssim \pi / d_{\text {phase }}$ and, therefore, is much smaller than the characteristic momenta, $p_{F}$ and $p_{T} \sim \sqrt{m T}$ for the degenerate and Boltzmann gas, respectively, Eq. (12) can be simplified as:

$$
\rho(x, t) \approx \rho_{0}+\int \frac{d p}{2 \pi} \widetilde{\rho}_{0}^{\prime}(p) \phi^{\prime}(x-(p / m) t),
$$

where $\rho_{0}=\int(d p /(2 \pi \hbar)) \widetilde{\rho}_{0}(p)$ is the initial constant density and $\widetilde{\rho}_{0}^{\prime}$ is the derivative of $\widetilde{\rho}_{0}$. The function $\phi^{\prime}(x)$ is only nonzero in the transition regions of the width $d_{\text {phase }}$. Therefore, Eq. (13) has a very clear meaning. It represents a superposition of bumps moving with different velocities $p / m$ which are distributed with the weights $\widetilde{\rho}_{0}^{\prime}(p)$.

For a degenerate Fermi gas at zero temperature one has $\widetilde{\rho}_{0}^{\prime}(p)=\delta\left(p+p_{F}\right)-\delta\left(p-p_{F}\right)$, and, hence,

$$
\rho(x, t) \approx \rho_{0}+\frac{1}{2 \pi}\left[\phi^{\prime}\left(x+\left(p_{F} / m\right) t\right)-\phi^{\prime}\left(x-\left(p_{F} / m\right) t\right)\right] .
$$

The above formula describes a "soliton-like" motion (without broadening and interaction) of bright and dark structures in the density, described by the function $\phi^{\prime}(x)$, in the opposite directions with the same velocity $v_{F}=$ $p_{F} / m$. The physical explanation of this phenomenon is very simple. Under the condition $d_{\text {phase }} \gg \lambda_{F}$, the quantum state of the system after the phase imprinting contains excitations, particle-hole pairs, only in the vicinity of the Fermi surface. (The energy width of the excitation distribution is of the order of $\hbar / d_{\text {phase }} \ll p_{F}$.) As a result, they have the same velocity. either $v_{F}$ or $-v_{F}$, and, therefore, the corresponding density profile moves in both directions with velocities $\pm v_{F}$, respectively, without any distortion. To be precise, the broadening in this case is of the second order in $d_{\text {phase }} / \lambda_{F}$. To determine the characteristic time scale $\tau_{F 0}$, one has to keep in mind that the width of the distribution of the excitation momenta after the phase imprinting is of the order 
of $\delta p \sim \hbar / d_{\text {phase }} \ll p_{F}$. Hence, the time $\tau_{F 0}$ can be estimated as $\tau_{F 0} \sim\left(\hbar / T_{F}\right)\left(d_{\text {phase }} / \lambda_{F}\right)^{2} \gg \hbar / T_{F}$.

At temperatures $T \ll T_{F}$ (but $T>T_{F} \lambda_{F} / d_{\text {phase }}$, the condition when the thermal width of the excitation distribution is larger then that after the phase imprinting), the situation is very similar, and Eq. (13) can be written in the form

$$
\begin{aligned}
\rho(x, t) \approx & \rho_{0}+\int \frac{d s}{2 \pi} f_{F}(s, T)\left[\phi^{\prime}\left(x+t\left(p_{F}+s\right) / m\right)\right) \\
& \left.-\phi^{\prime}\left(x-t\left(p_{F}+s\right) / m\right)\right],
\end{aligned}
$$

where $f_{F}^{\prime}(s, T)=\left(p_{F} / 4 m T\right) \cosh ^{-2}\left(s p_{F} / 2 m T\right)$ is related to the derivative of the Fermi-Dirac distribution and describes broadening of the $\delta$-like peaks at $p=$ $\pm p_{F}$ in the distribution $\widetilde{\rho}_{0}^{\prime}(p)$ at finite temperatures. As a result, now one has a superposition of bright bumps and dark wells in the density profile, moving with slightly different velocities distributed with two narrow peaks at $\pm p_{F} / m$ with the width of the order of $m T / p_{F} \sim p_{F} T / T_{F} \ll p_{F}$. This results in spreading of bumps and wells in the density profile on a time scale $\tau_{F T} \sim(\hbar / T)\left(d_{\text {phase }} / \lambda_{F}\right) \sim\left(\hbar / T_{F}\right)\left(T_{F} / T\right)\left(d_{\text {phase }} / \lambda_{F}\right) \gg$ $\hbar / T, \hbar / T_{F}$ (at $T=T_{F} \lambda_{F} / d_{\text {phase }}$ one has $\left.\tau_{F T}=\tau_{F 0}\right)$. Therefore, the time evolution of the gas density after a phase imprinting can be described in terms of a combination of several quasisolitons (bumps) and quasidarksolitons (wells) which move through each other without any interaction and, for times $t \ll \tau$, decay (broaden) very slowly. In Fig. 7 we present the results of numerical calculations of the time evolution of the density and Wigner functions for a spatially homogeneous gas at the temperature $T=0.01 T_{F}$ after the $2 \pi$-step phase imprinting with $d_{\text {phase }}=160 \lambda_{F}$. The results clearly

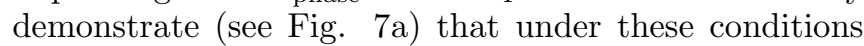
the soliton-like structures caused by the phase imprinting behave like real solitons, i.e., they propagate without any noticeable decay (broadening) on a long time scale. The blue regions in Figs. $7 \mathrm{~b}$ and $7 \mathrm{c}$ correspond to negative values of the Wigner function, and, therefore, indicate the quantum origin of the phenomenon.

For high temperatures $T \gg T_{F}$ gas is in the Boltzmann regime, and Eq. (13) takes the form

$$
\rho(x, t) \approx \rho_{0}+\int \frac{d p}{2 \pi} f_{B}(p, T) \phi^{\prime}(x-(p / m) t),
$$

where $f_{B}(p, t)=\rho_{0} \sqrt{2 \pi / m T}(p / m T) \exp \left(-p^{2} / 2 m T\right)$ describes the distribution with two broad peaks at $p= \pm p_{T}$ with $p_{T} \sim \sqrt{m T}$, with the width $\delta p \sim p_{T}$. In this case, the decay of the original density profile takes place more rapidly than in the case of a degenerate gas, on the time scale $\tau_{B} \sim(\hbar / T)\left(d_{\text {phase }} / \lambda_{T}\right) \sim \tau_{F} \sqrt{T_{F} / T} \gg \tau_{F}$. The corresponding numerical results for the density evolution at the temperature $T=5 T_{F}$ are shown in Fig. 8. Although the parameters of the phase imprinting are exactly the same as for the case of Fig. 7, the temperature
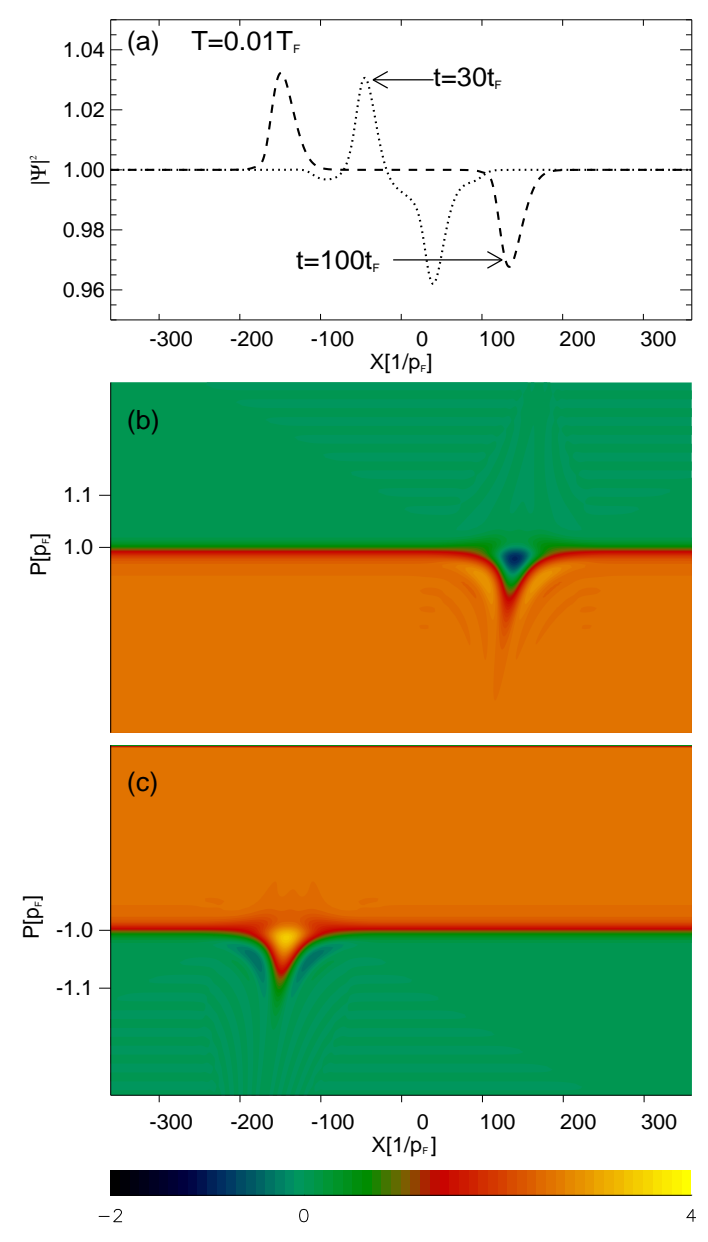

FIG. 7: The time evolution of the gas density and the Wigner function after the phase imprinting with $d_{\text {phase }}=160 \lambda_{F}$ at temperature $T=0.01 T_{F}$ : (a) the density profile at the times $t=30 t_{F}$ (the dotted curve) and $t=100 t_{F}$ (the dashed curve), respectively, where $t_{F}=\hbar / T_{F}$; (b) and (c) the Wigner function for the time $t=100 t_{F}$, where $t_{F}=\hbar / \varepsilon_{F}$.

(or, in other words, the absence of the sharp Fermi surface) cause a rapid decay of soliton-like structures.

The same consideration is applied to the trapped gas in the harmonic potential. In this case, due to the periodicity of the motion, the essential scale for the time evolution is set by the inverse level spacing: $t \leq 2 \pi / \omega$, where $\omega$ is the trap frequency. Therefore, after phase imprinting one would observe a quasisoliton behavior if $2 \pi / \omega \ll \tau_{F 0}, \tau_{F T}$, or $2 \pi / \omega \ll \tau_{B}$ for the degenerate or Boltzmann regime, respectively. More explicitly, in the degenerate regime one has the following requirement for the temperature $T$ and the width $d_{\text {phase }}:\left[\hbar \omega / \max \left(T, T_{F} \lambda_{F} / d_{\text {phase }}\right)\right]\left(d_{\text {phase }} / \lambda_{F}\right) \gg 1$, or $d_{\text {phase }} / l_{0} \gg \max (1, T / \hbar \omega \sqrt{N})$, where $l_{0}=\sqrt{\hbar / m \omega}$ is the oscillator length and $N$ is the number of particle. Correspondingly, in the regime of a Boltzmann gas, the requirement reads: $(\hbar \omega / T)\left(d_{\text {phase }} / \lambda_{T}\right) \gg 1$, or $d_{\text {phase }} / l_{0} \gg \sqrt{T / \hbar \omega}$. 


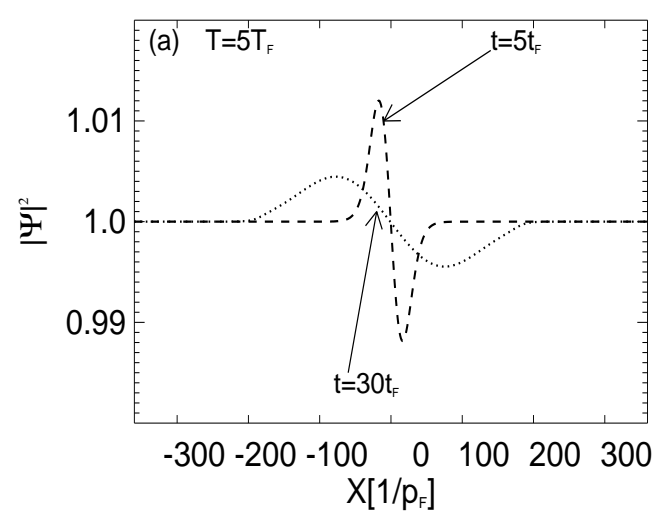

FIG. 8: The density of the gas with temperature $T=5 T_{F}$ at time $t=5 t_{F}$ (the dashed curve) and $t=30 t_{F}$ (the dotted curve), respectively, after the phase imprinting with $d_{\text {phase }}=$ $160 \lambda_{F}$.

In the regime where $d_{\text {phase }} \lesssim \lambda_{F}, \lambda_{T}$, the excitations created during the phase imprinting have a very broad distribution with the width $\delta p \sim \hbar / d_{\text {phase }} \gtrsim$ $p_{F}, p_{T}$ in a spatially homogeneous gas, and $\delta \varepsilon \sim$ $\hbar \omega\left(l_{0} / d_{\text {phase }}\right)^{2}$ in a trapped one. In this case one would expect a very rapid decay of "solitons" on a time scale $\tau \sim\left[\hbar / \max \left(T_{F}, T\right)\right]\left[d_{\text {phase }} / \min \left(\lambda_{F}, \lambda_{T}\right)\right]^{2} \ll \hbar / T_{F}$ for both spatially homogeneous and trapped Fermi gases. The corresponding numerical results for the density and Wigner function evolution after the $2 \pi$-step phase imprinting with $d_{\text {phase }}=2 \lambda_{F}$ are shown in Figs. 9 (for the temperature $T=0.01 T_{F}$ ) and 10 (for the temperature $T=T_{F}$ ). In the quantum degenerate case (Fig. 9), the Wigner function has a well pronounced fringes structure, and the corresponding complicated structure in the density profile decays rather fast. For the non-degenerate gas (Fig. 10), the Wigner function is smooth everywhere except for the narrow region subjected by the phase imprinting. In this case, the density profile has no smallscale structures, as compared to the degenerate case from Fig. 9, but also relaxes very fast to the uniform density distribution.

\section{DENSITY MATRIX FORMALISM}

Another way to discuss the soliton's formation in a gas of neutral fermionic atoms is to start from a set of equations for reduced density matrices. In particular, the equation of motion for the one-particle density matrix involves the two-particle density matrix $\rho_{2}$ and is given by $[17$

$$
\begin{aligned}
& i \hbar \frac{\partial}{\partial t} \rho_{1}\left(\vec{r}_{1}, \vec{r}_{2}, t\right)=-\frac{\hbar^{2}}{2 m}\left(\vec{\nabla}_{1}^{2}-\vec{\nabla}_{2}^{2}\right) \rho_{1}\left(\vec{r}_{1}, \vec{r}_{2}, t\right) \\
& +\int d^{3} r^{\prime}\left[V\left(\vec{r}_{1}-\vec{r}^{\prime}\right)-V\left(\vec{r}_{2}-\vec{r}^{\prime}\right)\right] \rho_{2}\left(\vec{r}_{1}, \vec{r}^{\prime} ; \vec{r}_{2}, \vec{r}^{\prime}, t\right)
\end{aligned}
$$
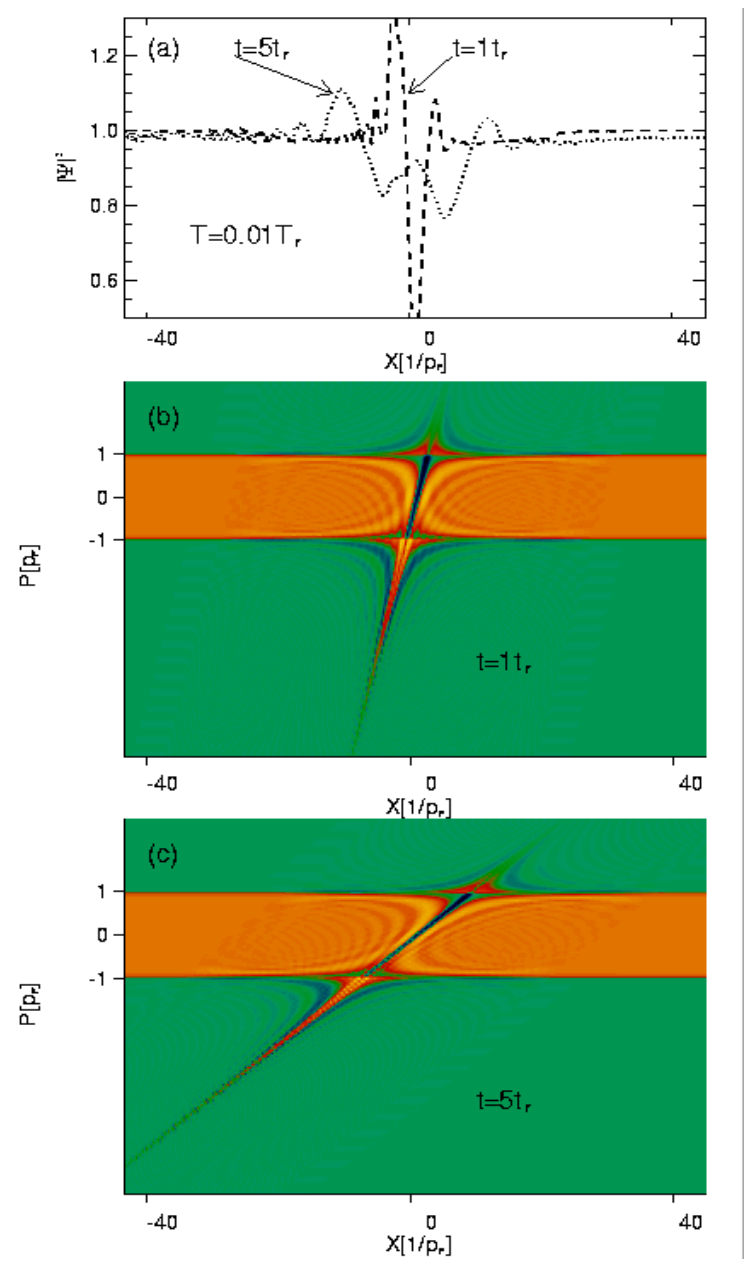

FIG. 9: The time evolution of the gas density and the Wigner function after the phase imprinting with $d_{\text {phase }}=2 \lambda_{F}$ : (a) the gas density at the times $t=t_{F}$ (the dashed curve) $t=5 t_{F}$ (the dotted curve); (b) the Wigner function at the time $t=t_{F}$; (c) the Wigner function at the time $t=5 t_{F}$. The temperature of the gas $T=0.01 T_{F}$.

$$
+\left[V_{e x t}\left(\vec{r}_{1}, t\right)-V_{e x t}\left(\vec{r}_{2}, t\right)\right] \rho_{1}\left(\vec{r}_{1}, \vec{r}_{2}, t\right),
$$

where $V\left(\vec{r}_{1}-\vec{r}_{2}\right)$ is the two-particle interaction term and $V_{\text {ext }}(\vec{r}, t)$ is the external potential. In the limit $\vec{r}_{1} \rightarrow \vec{r}_{2}$ Eq. (17) leads to the continuity equation

$$
\frac{\partial \rho(\vec{r}, t)}{\partial t}+\vec{\nabla} \cdot[\rho(\vec{r}, t) \vec{v}(\vec{r}, t)]=0,
$$

where the density and velocity fields are defined as follows:

$$
\begin{aligned}
\rho(\vec{r}, t) & =\lim _{\vec{r}_{1} \rightarrow \vec{r}_{2}} \rho_{1}\left(\vec{r}_{1}, \vec{r}_{2}, t\right) \\
\vec{v}(\vec{r}, t) & =\frac{\hbar}{2 m} \lim _{\vec{r}_{1} \rightarrow \vec{r}_{2}}\left(\vec{\nabla}_{1}-\vec{\nabla}_{2}\right) \chi_{1}\left(\vec{r}_{1}, \vec{r}_{2}, t\right)
\end{aligned}
$$

and $\chi_{1}\left(\vec{r}_{1}, \vec{r}_{2}, t\right)$ is the phase of the one-particle density matrix.

One can also rewrite Eq. (17) introducing the centerof-mass $\left(\vec{R}=\left(\vec{r}_{1}+\vec{r}_{2}\right) / 2\right)$ and the relative position $(\vec{s}=$ 

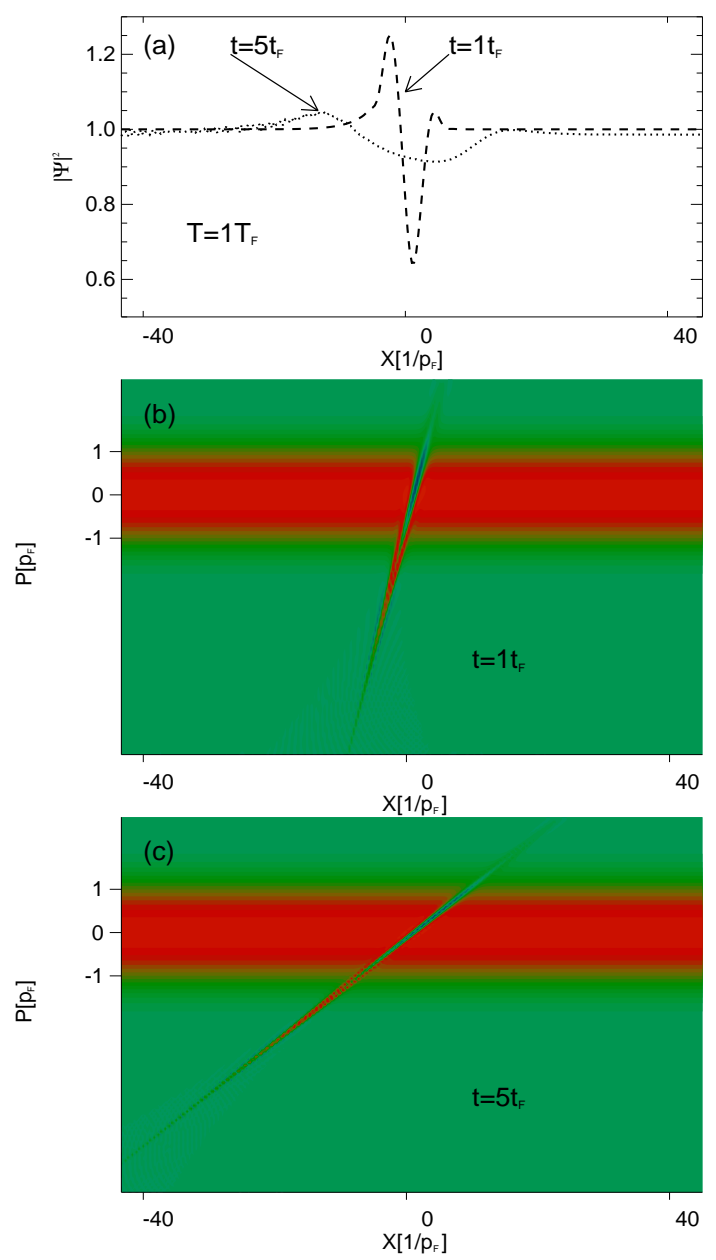

FIG. 10: The same as in Fig. 6 but for the temperature $T=T_{F}$.

$\left.\vec{r}_{1}-\vec{r}_{2}\right)$ coordinates. By taking the derivative of Eq. (17) with respect to the coordinate $\vec{s}$ the hydrodynamic Euler-type equation of motion is obtained in the limit $\vec{s} \rightarrow 0$

$$
\begin{aligned}
\frac{\partial \vec{v}(\vec{r}, t)}{\partial t} & =-\frac{\vec{\nabla} \cdot \mathrm{T}}{m \rho(\vec{r}, t)}-[\vec{v}(\vec{r}, t) \cdot \vec{\nabla}] \vec{v}(\vec{r}, t) \\
& +\frac{\vec{F}_{\text {int }}(\vec{r}, t)}{m \rho(\vec{r}, t)}-\frac{\vec{\nabla} V_{\text {ext }}(\vec{r}, t)}{m} .
\end{aligned}
$$

Here, the kinetic-energy stress tensor T, whose elements are given by

$$
\mathrm{T}_{k l}=-\frac{\hbar^{2}}{m} \lim _{\vec{s} \rightarrow 0} \frac{\partial^{2} \sigma_{1}(\vec{R}, \vec{s}, t)}{\partial s_{k} \partial s_{l}},
$$

depends on $\sigma_{1}\left(\vec{r}_{1}, \vec{r}_{2}, t\right)$, the amplitude of one-particle density matrix and can be calculated based on a local equilibrium assumption which is the substance of Thomas-Fermi approximation. $\vec{F}_{\text {int }}(\vec{r}, t)$, on the other hand, represents the force due to fermion-fermion interaction (for spin-polarized atomic fermions the dipole- dipole interaction is a good candidate) and is defined as

$$
\vec{F}_{\text {int }}(\vec{r}, t)=-\int d^{3} r^{\prime} \vec{\nabla}_{\vec{r}} V\left(\vec{r}-\vec{r}^{\prime}\right) \rho_{2}\left(\vec{r}, \vec{r}^{\prime} ; \vec{r}, \vec{r}^{\prime}, t\right)
$$

The one-particle Wigner function within the ThomasFermi approximation is given by

$$
w(\vec{r}, \vec{p})=\eta\left(\hbar^{2}\left[6 \pi^{2} \rho(\vec{r})\right]^{2 / 3}-\vec{p}^{2}\right),
$$

where $\eta()$ is Heaviside's unit step function and hence the one-particle density matrix is calculated as

$$
\rho_{1}(\vec{R}, \vec{s})=\int \frac{d^{3} p}{(2 \pi \hbar)^{3}} w(\vec{R}, \vec{p}) e^{i \vec{p} \vec{s} / \hbar} .
$$

After straightforward algebra one obtains:

$\rho_{1}(\vec{R}, \vec{s})=\frac{2}{(2 \pi \hbar)^{2}} \frac{1}{s}\left[-\frac{\hbar p_{F}}{s} \cos \left(\frac{p_{F} s}{\hbar}\right)+\frac{\hbar^{2}}{s^{2}} \sin \left(\frac{p_{F} s}{\hbar}\right)\right]$.

The one-particle density matrix is a real function in this case and depends only on the length $s=|\vec{s}|$ of vector $\vec{s}$. In the limit $\vec{s} \rightarrow 0$ the kinetic-energy stress tensor $\mathrm{T}$ is getting diagonal with $\left(\hbar^{2} / m\right)\left(1 / 30 / \pi^{2}\right)\left(6 \pi^{2} \rho\right)^{5 / 3}$ term at each position. Then, assuming the case of noninteracting fermions (spin-polarized atoms at low temperature), the Eqs. (18) and (20) become a closed set of equations for the density and velocity fields:

$$
\begin{aligned}
& \frac{\partial \rho}{\partial t}+\vec{\nabla} \cdot(\rho \vec{v})=0 \\
& \frac{\partial \vec{v}}{\partial t}+(\vec{v} \cdot \vec{\nabla}) \vec{v}+\vec{\nabla}\left(\frac{\hbar^{2}}{2 m^{2}}\left(6 \pi^{2}\right)^{3 / 2} \rho^{2 / 3}+\frac{V_{\text {ext }}}{m}\right)=0,
\end{aligned}
$$

Note that the Eqs. (18) and (20) are general and could be used also for a bosonic system. For example, for the system of bosons described by the many-body wave function $\Psi\left(\vec{r}_{1}, \ldots, \vec{r}_{N}\right)=\varphi\left(\vec{r}_{1}\right) \cdot \ldots \cdot \varphi\left(\vec{r}_{N}\right)$ (as in the case of the Bose-Einstein condensate in the mean-field approximation) the one-particle density matrix is given by

$$
\rho_{1}(\vec{R}, \vec{s})=\varphi\left(\vec{R}+\frac{1}{2} \vec{s}\right) \varphi^{*}\left(\vec{R}-\frac{1}{2} \vec{s}\right) .
$$

Now, the kinetic-energy stress tensor possesses offdiagonal elements and leads to the so-called "quantum pressure" term in the equation of motion. Assuming contact interaction between the bosonic atoms dominates, the appropriate equations are written as

$$
\begin{aligned}
& \frac{\partial \rho}{\partial t}+\vec{\nabla} \cdot(\rho \vec{v})=0 \\
& \frac{\partial \vec{v}}{\partial t}+\vec{\nabla}\left(\frac{4 \pi \hbar^{2} a}{m^{2}} \rho+\frac{\vec{v}^{2}}{2}+\frac{V_{\text {ext }}}{m}-\frac{\hbar^{2}}{2 m^{2}} \frac{\vec{\nabla}^{2} \sqrt{\rho}}{\sqrt{\rho}}\right)=0
\end{aligned}
$$

and are the hydrodynamic [18] representation of the Gross-Pitaevskii equation. 
It is easy to check that for the spatially homogeneous system, the one-dimensional counterpart of Eqs. (22) has the following solution

$$
\begin{aligned}
& \rho(x, t)=\rho_{0}+\frac{1}{2 \pi}\left[\phi^{\prime}\left(x+\left(p_{F} / m\right) t\right)-\phi^{\prime}\left(x-\left(p_{F} / m\right) t\right)\right] \\
& v(x, t)=-\frac{\hbar}{2 m}\left[\phi^{\prime}\left(x+\left(p_{F} / m\right) t\right)+\phi^{\prime}\left(x-\left(p_{F} / m\right) t\right)\right]
\end{aligned}
$$

assuming the slope of the phase being imprinted is much less than the unperturbed density $\left(\phi^{\prime} \ll \rho_{0}\right)$. This assumption is equivalent to the condition $d_{\text {phase }} \gg \lambda_{F}$ considered by us in Sec. III and the solution (24) coincides with expression (14). It also coincides with the Thomas-Fermi approximation, valid in one-dimensional space when the number of atoms is large enough (Sec. II). Again, the solution (24) represents two quasisolitons (a bright and a dark one) traveling in opposite directions after imprinting (at $t=0$ ) the phase $\phi(x)$. The speed of quasisolitons is equal to the speed of sound. A similar solution can be found also in two- and three-dimensional case

$$
\begin{aligned}
\rho(t) & =\rho_{0}+\alpha\left[\phi^{\prime}(x+c t)-\phi^{\prime}(x-c t)\right] \\
v_{x}(t) & =-\frac{\hbar}{2 m}\left[\phi^{\prime}(x+c t)+\phi^{\prime}(x-c t)\right],
\end{aligned}
$$

where $c$ equals the speed of sound, $\alpha \sim \rho_{0}^{1 / 2}$ or $\alpha \sim \rho_{0}^{2 / 3}$ respectively in two- and three-dimensional space, and the phase being imprinted changes only in ' $x$ ' direction. Again, the solutions (25) are valid in the limit $d_{\text {phase }} \gg$ $\lambda_{F}$.

It is clear from Fig. 3 that in the regime where $d_{\text {phase }} \lesssim \lambda_{F}$, the dynamics of fermionic system should be discussed rather in terms of soliton-like structures with dispersion leading to the fast broadening of pulses. Even at zero temperature the phase imprinting excites states with energy above Fermi level, depending on the width of the phase step $d_{\text {phase }}$. The deviation of the group velocity of bright and dark wave packets from the zerotemperature sound velocity $p_{F} / m$ maybe qualitatively understood by the following argument. In the spirit of the local density approximation one considers local value of the Fermi momentum determined by the local value of the density. Since the local density is lower at the center of dark quasisoliton its velocity is thus reduced. Of course, the opposite is true for the bright quasisoliton. Using Eq. (24) one gets local corrections to $p_{F}$ of the form $\delta p_{F}=\mp \frac{\hbar}{2} \phi^{\prime}(0)$. This correction agrees with numerical results in the order of magnitude. More precise estimation of the asymmetry of velocities requires, however, taking into account dispersion effects and systematic expansion in $\lambda_{F} / d_{\text {phase }}$ up to higher orders.

There is an equivalent way of calculating the kineticenergy stress tensor $\mathrm{T}$ (21), involving the one-particle Wigner function

$$
\mathrm{T}_{k l}=\int \frac{p_{k} p_{l}}{m} w(\vec{r}, \vec{p}) d^{3} p
$$

which is, however, more suitable for the finitetemperature extension of the Thomas-Fermi approach. In equilibrium, the Wigner function for a uniform system is just the Fermi-Dirac distribution function $f(\varepsilon)=$ $(\exp (\beta(\varepsilon-\mu))+1)^{-1}$ with $\varepsilon=p^{2} / 2 m$ and $\mu$ being the particle's kinetic energy and the chemical potential (constant for a homogeneous case) respectively. The ThomasFermi approximation assumes, as usually, that the system is locally uniform and the appropriate equations (in one-dimensional form) are written as

$$
\begin{aligned}
& \frac{\partial \rho}{\partial t}+\frac{\partial}{\partial x}(\rho v)=0 \\
& \frac{\partial v}{\partial t}+v \frac{\partial v}{\partial x}+\frac{1}{m \rho} \frac{\partial}{\partial x} \frac{1}{2 \pi \hbar} \int_{-\infty}^{\infty} \frac{p^{2} / m d p}{\exp \left(\beta\left(\frac{p^{2}}{2 m}-\mu\right)\right)+1}=0 .
\end{aligned}
$$

Here, the chemical potential is position-dependent and is calculated from the relation

$$
\rho=\frac{1}{2 \pi \hbar} \int_{-\infty}^{\infty} \frac{d p}{\exp \left(\beta\left(\frac{p^{2}}{2 m}-\mu\right)\right)+1} .
$$

We consider now the case when the temperature is finite but the gas is still strongly degenerate, i.e. $T \ll$ $T_{F}$. By using the Sommerfeld expansion and keeping only the first term one can easily find the expression $\mu=(2 \pi \hbar)^{2} /(8 m) \rho^{2}$. It turns out that in the regime where the imprinted phase changes slowly in comparison with unperturbed density, the finite-temperature timedependent Thomas-Fermi model defined by the Eqs. (27) and (28) has the following solution

$$
\begin{aligned}
& \rho=\rho_{0}+\int_{0}^{\infty} \frac{d \varepsilon}{2 \pi}\left(-\frac{\partial f}{\partial \varepsilon}\right)\left[\phi^{\prime}\left(x+\sqrt{\frac{2 \varepsilon}{m}} t\right)-\phi^{\prime}\left(x-\sqrt{\frac{2 \varepsilon}{m}} t\right)\right] \\
& v=-\frac{\hbar}{2 m} \int_{0}^{\infty} d \varepsilon\left(-\frac{\partial f}{\partial \varepsilon}\right)\left[\phi^{\prime}\left(x+\sqrt{\frac{2 \varepsilon}{m}} t\right)+\phi^{\prime}\left(x-\sqrt{\frac{2 \varepsilon}{m}} t\right)\right]
\end{aligned}
$$

For the temperature $T=0$ one has $(-\partial f / \partial \varepsilon)=$ $\delta\left(\varepsilon-\varepsilon_{F}\right)$ and the solution (29) reduces to the one found previously (14, 24). To verify the ansatz (29) we put it in the set of Eqs. (27) and keep only linear terms in $\phi^{\prime}$ (according to $d_{\text {phase }} \gg \lambda_{F}$ ). The solvability condition has then the form

$$
\begin{aligned}
& \int_{0}^{\infty} d \varepsilon\left(-\frac{\partial f}{\partial \varepsilon}\right)\left[\sqrt{\frac{2 \varepsilon}{m}}-\sqrt{\frac{2 \varepsilon_{F}}{m}}\right] \times \\
& {\left[\phi^{\prime \prime}\left(x+\sqrt{\frac{2 \varepsilon}{m}} t\right) \pm \phi^{\prime \prime}\left(x-\sqrt{\frac{2 \varepsilon}{m}} t\right)\right]=0}
\end{aligned}
$$

and is true for low temperatures $T \ll T_{F}$ since then the derivative of the Fermi-Dirac distribution function 
TABLE II: Speeds of dark and bright quasisolitons (in units of speed of sound) generated in a one-dimensional homogeneous Fermi gas of various number of atoms after imprinting a single phase step of $\pi$ and the width of $2.5 \lambda_{F}$

\begin{tabular}{rcc}
\hline \hline $\mathrm{N}$ & $\frac{v_{b}}{c}$ & $\frac{v_{d}}{c}$ \\
\hline 500 & -1.061 & +0.937 \\
1000 & -1.038 & +0.965 \\
5000 & -1.010 & +0.993 \\
8000 & -1.006 & +0.994 \\
\hline \hline
\end{tabular}

strongly peaks at $\varepsilon=\varepsilon_{F}$, whereas the rest of the integrand is very small. The solution (29) is identical with (15).

\section{A. Numerical results}

We have solved numerically the set of Eqs. (22). To this end, we applied the inverse Madelung transformation [19] to Eqs. (22) and used the Split-Operator technique. Imaginary-time propagation method was chosen to generate the ground state of a nonuniform (trapped) Fermi gas. Real-time propagation, on the other hand, allows to investigate the phase imprinting and developing instabilities after that.

First, by imprinting the phase on a one-dimensional uniform system we again investigate the regime where $d_{\text {phase }} \gg \lambda_{F}$, however, this time by increasing the number of atoms while keeping the same the width of the phase step. Of course, larger number of atoms means smaller Fermi length. Increasing the number of atoms is easily attainable within Thomas-Fermi approach as opposed to the direct solution of the manybody Schrödinger equation. Results regarding the speeds of bright and dark quasisolitons are presented in Table II. It is clear that reaching the above mentioned regime forces both quasisolitons to move with the same velocity equal to the speed of sound.

\section{CONCLUSIONS}

In this paper we have studied in detail the possibility of generation of soliton-like structures in ultracold Fermi gases using the method of phase imprinting. Generation of such structures is feasible with existing experimental setups. The soliton-like structures are generated because in the process of phase imprinting in the limit in which the characteristic length of the imprint $d_{\text {phase }} \ll \lambda_{F}$ only a narrow band of states with momenta close to $\pm p_{F}$ are excited. The life time of soliton-like structures can be quite long even at not extremely low temperatures allowing for observations. We have presented both numerical results and analytic theory explaining the mechanism of generation and evolution of soliton-like structures both in homogeneous quasi 1D cylinders and in quasi 1D harmonic traps. It will be interesting to generalize the presented theory to the case of two-component Fermi gas in normal phase.

\section{Acknowledgments}

We thank C. Salomon, J. Dalibard, L. Santos, A. Sanpera. This paper has been supported by Deutsche Forschungsgemeinschaft (among others in the frame of SFB 407, SP 1116, GK 282, and 436POL), TRN 'Cold Atoms and Molecules', ESF PESC Programm 'BEC 2000+'. K.R. acknowledges support of the Subsidy by Foundation for Polish Science. Part of the results has been obtained using computers at the Interdisciplinary Center for Mathematical and Computational Modeling at Warsaw University.
[1] B. DeMarco and D.S. Jin, Science 285, 1703 (1999).

[2] B. DeMarco, S.B. Papp, and D.S. Jin, Phys. Rev. Lett. 86, 5409 (2001).

[3] A.G. Truscott, K.E. Strecker, W.I. McAlexander, G.B. Partridge, and R.G. Hulet, Science 291, 2570 (2001).

[4] F. Schreck, L. Khaykovich, K.L. Corwin, G. Ferrari, T. Bourdel, J. Cubizolles, and C. Salomon, Phys. Rev. Lett. 87, 080403 (2001).

[5] D.A. Butts and D.S. Rokhsar, Phys. Rev. A 55, 4346 (1997).

[6] G.M. Bruun and K. Burnett, Phys. Rev. A 58, 2427 (1998).

[7] R. Roth and H. Feldmeier, cond-mat/0108255.

[8] G.M. Bruun and C.W. Clark, Phys. Rev. Lett. 83, 5415 (1999).

[9] L. Vichi and S. Stringari, Phys. Rev. A 60, 4734 (1999).

[10] K. Góral, B.-G. Englert, and K. Rzążewski, Phys. Rev.
A 63, 033606 (2001).

[11] T. Karpiuk, M. Brewczyk, and K. Rzążewski, condmat/0103564

12 B. Damski, K. Sacha, and J. Zakrzewski, condmat/0111236.

13] S. Burger, S. Dettmer, W. Ertmer, K. Sengstock, A. Sanpera, G.V. Shlyapnikov, and M. Lewenstein, Phys. Rev. Lett. 83, 5198 (1999).

[14] J. Denschlag, J.E. Simsarian, D.L. Feder, C.W. Clark, L.A. Collins, J. Cubizolles, L. Deng, E.W. Hagley, K. Helmerson, W.P. Reinhardt, S.L. Rolston, B.I. Schneider, W.D. Phillips, Science 287, 97 (2000).

[15] Ł. Dobrek, M. Gajda, M. Lewenstein, K. Sengstock, G. Birkl, and W. Ertmer, Phys. Rev. A 60, R3381 (1999).

[16] G. Andrelczyk, M. Brewczyk, Ł. Dobrek, M. Gajda, and M. Lewenstein, Phys. Rev. A 64, 043601 (2001).

[17] H. Frölich, Physica 37, 215 (1967). 
[18] E. Madelung, Z. Phys. 40, 322 (1926).

[19] B.Kr. Dey and B.M. Deb, Int. J. Quantum Chem. 70, 441 (1998); A. Domps, P.-G. Reinhard, and E. Suraud,

Phys. Rev. Lett. 80, 5520 (1998). 